\title{
Deep vacancy induced low-density fluxional interfacial water
}

\author{
Keyang Liu $\odot$ \\ School of Physics, Peking University, Beijing 100871, People's Republic of China \\ Jianqing Guo \\ International Center for Quantum Materials, School of Physics, Peking University, Beijing 100871, People's Republic of China \\ Weizhong Fu $\odot$ \\ School of Physics, Peking University, Beijing 100871, People's Republic of China \\ Ji Chen $\odot^{*}$ \\ School of Physics, Peking University, Beijing 100871, People's Republic of China; \\ Collaborative Innovation Center of Quantum Matter, Beijing 100871, People's Republic of China; \\ Interdisciplinary Institute of Light-Element Quantum Materials and Research Center for Light-Element Advanced Materials, Peking \\ University, Beijing 100871, People's Republic of China
}

(Received 5 July 2021; revised 7 September 2021; accepted 19 October 2021; published 29 October 2021)

\begin{abstract}
Interfacial water on transition metal oxides such as $\mathrm{TiO}_{2}$ has been widely studied because of its structural complexity and scientific relevance in, e.g., photocatalysis and ice growth. Using $a b$ initio molecular dynamics, we find that interfacial water on the anatase (101) surface features an unconventional fluxional structure with reduced contact layer density. The density reduction and flexibility of interfacial water are induced by oxygen vacancy defects located deep below the surface. Our study proposes a fresh perspective of the anatase-water interface, raising the importance of nontrivial long-range effects caused by deep defects. These often-neglected effects highlight the necessity and challenges of the state-of-the-art simulation and experimental probing of solid-liquid interfaces.
\end{abstract}

DOI: 10.1103/PhysRevResearch.3.L042014

Water is one of the most ubiquitous materials in nature and has an incredibly diverse range of structures and properties at the interfaces with other materials [1-3]. Aqueous interfaces are fundamental to many natural phenomena and modern technologies [4-6]. Understanding the molecular structure of interfacial liquid water is key to examining the physical and chemical processes at interfaces. For example, the ordering and density of interfacial water are two key elements for heterogeneous ice crystallization [5,7], electric double layers formed at water/solid interfaces control many electrochemical reactions [8-11], and interfacial water crucially affects energy converting in modern nanotechnologies [12-15]. Because of these important applications, many experimental studies have been carried out to establish microscopic structure of interfacial water on surfaces, including, e.g., high-resolution imaging in vacuum [16], in situ diffraction measurements [17], and vibrational spectroscopy $[18,19]$. Molecular dynamics simulations based on density

\footnotetext{
*ji.chen@pku.edu.cn

Published by the American Physical Society under the terms of the Creative Commons Attribution 4.0 International license. Further distribution of this work must maintain attribution to the author(s) and the published article's title, journal citation, and DOI.
}

functional theory and force fields have also been very successful in elucidating interfacial structure and water dissociation [2,20-23].

From a different perspective, understanding the details of substrate materials can lead to fine-tuning of properties at solid-liquid interfaces [2]. For example, in titanium dioxide, a material that has been widely investigated for photocatalysis, reduced defects such as oxygen vacancies are considered to be ubiquitous $[24,25]$. Studies have shown that defects on the surface can react with water and subsurface defects can migrate to the surface to interact with water [26-28]. In addition, excess electrons induced by defects can also migrate and affect water adsorption and dissociation [13,29-31]. These interfacial effects, involving direct chemical interactions between the water and surface, are intuitively understandable [25]. In contrast, long-range physical effects induced by defects are generally less important. However, the fact that there is a delicate balance between water adsorption and the hydrogen bonding network suggests interfacial water properties may be sensitive to deep defects below the surface as well.

In this study we investigate the sensitivity of the interfacial water structure to oxygen vacancy, a natural defect in $\mathrm{TiO}_{2}$. We find that oxygen vacancies, surprisingly even very deep vacancies, have strong impacts on the structures of interfacial water. The impacts feature a density reduction and a fluxional behavior of interfacial water. These effects are not a direct 
consequence of reactions with surface oxygen vacancies and polarons, but rather due to a delicate balance of (i) hydrogen bonding between water molecules, (ii) adsorption of water, (iii) hydrogen bonding interactions between water and the substrate, and (iv) long-range electrostatic interactions.

Ab initio molecular dynamics simulations [32] were carried out using density functional theory (DFT) with the Vienna ab initio simulation package [33]. The van der Waals inclusive optB86b-vdW exchange correlation functional was employed $[34,35]$. Additional simulations with the strongly constrained and appropriately normed (SCAN)-revised Vydrov-van Voorhis (rVV10) exchange correlation functional [36,37] and with Hubbard corrections $(U=3.9 \mathrm{eV})[38]$ were performed to test the validity of conclusions. Electron-ion interactions were described using the projector augmented wave method, with $\mathrm{Ti}(3 d, 4 s), \mathrm{O}(2 s, 2 p)$, and $\mathrm{H}(1 s)$ electrons treated explicitly as valence electrons [39]. Wave functions were expanded in plane waves up to a kinetic energy cutoff of $500 \mathrm{eV}$. The $K$-point sampling was restricted to the $\Gamma$ point only. The deuterium mass ( $2 \mathrm{amu}$ ) was used for hydrogen to allow for a larger time step of $1 \mathrm{fs}$. Temperature was controlled using a Nosé-Hoover thermostat at $330 \mathrm{~K}$ within the $N V T$ ensemble. Ten different configurations were considered for anatase (101), including a stoichiometric substrate and nine substrates, each containing one oxygen vacancy $\left(V_{\mathrm{O}}\right)$ located at different depths. The oxygen vacancy concentrations in our supercell were $\frac{1}{120}$ and $0.9 \mathrm{~nm}^{-2}$, which are within the typical ranges in reduced $\mathrm{TiO}_{2}$ [40]. Taking the stoichiometric configuration as an example, a periodically repeated $1 \times 3$ supercell of size $10.21 \times 11.328 \times 31.58 \AA^{3}$ was used in our simulation, corresponding to a composition of ten Ti layers and twenty $\mathrm{O}$ layers $\left(\mathrm{Ti}_{60} \mathrm{O}_{120}\right)$ and a (101) surface area of $10.21 \times 11.328 \AA^{2}$. Water slabs including 52 water molecules were constructed with the bulk water density $\left(1.02 \mathrm{~g} / \mathrm{cm}^{3}\right)$. After reaching equilibrium in, e.g., $10 \mathrm{ps,} \mathrm{trajectories} \mathrm{were}$ collected for up to $120 \mathrm{ps}$. The pressure was within $50 \mathrm{MPa}$ throughout the simulations. We carried out additional simulations with $15 \AA$ vacuum above 76 water molecules and a substrate with $36 \mathrm{Ti}$ atoms and 71 (72) oxygen atoms [the $V_{\mathrm{O}} 8$ (pristine) system]. The bottom layer of the substrate was fixed to keep the bulk $\mathrm{TiO}_{2}$ properties (see Fig. S15 in [41]). We also tested other models, including anatase $(001)\left(\mathrm{Ti}_{90} \mathrm{O}_{180}\right)$, rutile (110) $\left(\mathrm{Ti}_{64} \mathrm{O}_{128}\right)$, and silica (0001) $\left(\mathrm{Si}_{24} \mathrm{O}_{48}\right)$. Further details are described in the Supplemental Material [41].

The anatase (101) surface has a sawtoothlike structure composed of both fivefold coordinated titanium $\left(\mathrm{Ti}_{5 c}\right)$ atoms and twofold coordinated oxygen $\left(\mathrm{O}_{2 c}\right)$ atoms along the [010] direction, as highlighted in Fig. 1. Compared with their coordination number in the bulk, $\mathrm{O}_{2 c}$ and $\mathrm{Ti}_{5 c}$ are undercoordinated and easily capture water. Previous studies of water adsorption on the anatase (101) surface in ultrahigh vacuum have suggested that both $\mathrm{Ti}_{5 c}$ and $\mathrm{O}_{2 c}$ are favorable bonding sites, presenting two ordered layers of water. This structure of water overlayer was also reproduced in many previous simulations [42-45]. For the liquid water-anatase interface at room temperature, however, the structure is not clear [46].

Figures 1(a) and 1(b) show an interfacial water structure observed in the simulation on the pristine substrate. The structure of the contact water layer resembles the overlayer structure on the anatase (101) surface in vacuum, containing
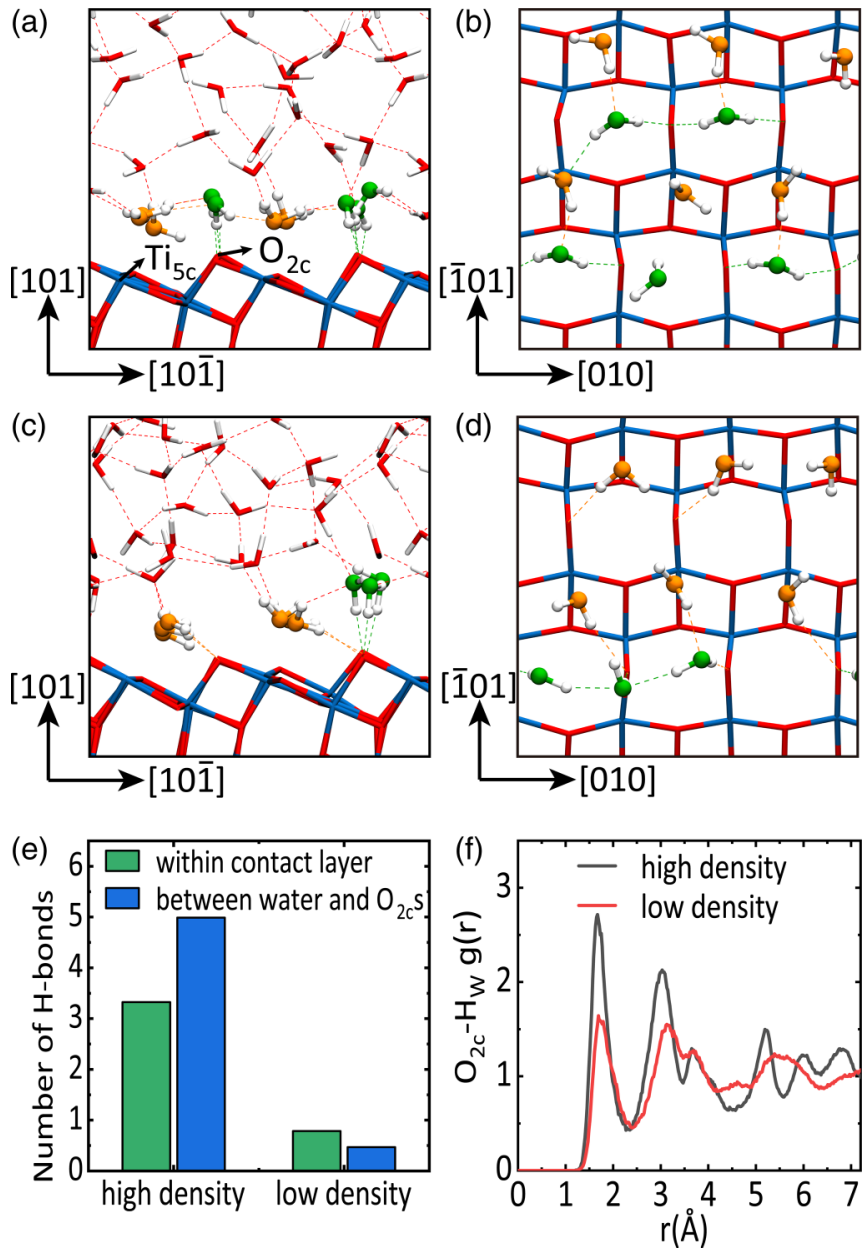

FIG. 1. Comparison of the low-density and the high-density interfacial water. (a) Side view and (b) top view of the high-density interfacial water. The oxygen and titanium atoms are represented by red and blue, respectively. Orange and green spheres highlight water molecules in the contact layer on $\mathrm{Ti}_{5 c}$ and $\mathrm{O}_{2 c}$ sites, respectively. (c) Side view and (d) top view of the low-density interfacial water. (e) Number of hydrogen bonds formed in two density models. Green bars correspond to hydrogen bonds between water molecules within the contact layer and blue bars correspond to hydrogen bonds formed between $\mathrm{O}_{2 c}$ and water. (f) Radial distribution function of $\mathrm{O}_{2 c}-\mathrm{H}_{W}$ pairs, where $\mathrm{H}_{W}$ is the hydrogen atom in liquid water.

alternating rows of adsorbed water. One row is along the $\mathrm{Ti}_{5 c}$ sites (adsorbed water highlighted in yellow) and the other is along the $\mathrm{O}_{2 c}$ sites (adsorbed water highlighted in green). Water molecules bonded to $\mathrm{O}_{2 c}$ mainly form donating hydrogen bonds with neighboring $\mathrm{O}_{2 c}$ sites and accepting hydrogen bonds with water molecules on $\mathrm{Ti}_{5 c}$. Because the structure contains two bonded water molecules per Ti site, effectively covering all surface sites, we refer to it as high-density interfacial water. ${ }^{1}$

\footnotetext{
${ }^{1}$ The density of interfacial water cannot be directly compared with bulk water because of the arbitrariness of the choice of the interfacial regime. However, for a rough comparison the lateral density of the high-density interfacial water on anatase (101) is slightly lower than
} 


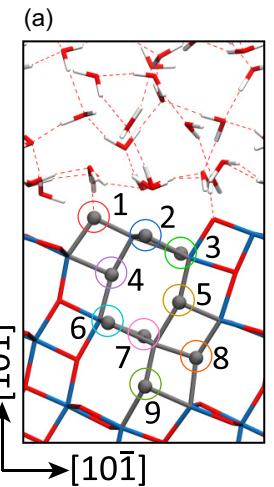

(b)

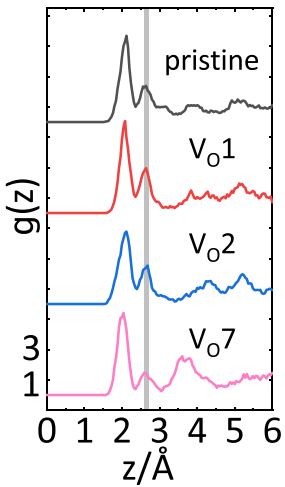

(c)

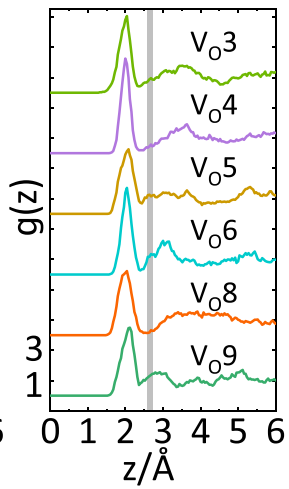

FIG. 2. Structural model and $g(z)$ profiles of ten systems. (a) Side view of a snapshot from the simulation using the pristine substrate. The nine oxygen vacancy sites of defective systems are labeled with numbers 1 to 9 corresponding to the first to the ninth oxygen layer under the surface. The $g(z)$ profiles for all systems are classified as (b) high-density interfacial water and (c) low-density interfacial water. The colors and labels are consistent with those in (a). Here $g(z)=\frac{N_{z, z+\Delta(z)}}{N_{\text {total }}}$, where $N_{z, z+\Delta(z)}$ is the number of water molecules within a $\Delta(z)$ thick slab and $N_{\text {total }}$ is the total number of water molecules. The reference point $z=0$ is chosen as the average $z$ value of all $\mathrm{Ti}_{5 c}$ sites of the whole trajectory. In each $g(z)$ profile, the reference point $z=0$ is chosen as the average $z$ value of all $\mathrm{Ti}_{5 c}$ sites of the whole trajectory. The vertical gray line indicates the position of the second peak on $g(z)$ profiles of the conventional high-density interfacial water. The interfacial water density integrated from $g(z)$ profiles is shown in Fig. 3(c). The convergence tests are discussed in Figs. S1 and S2 in [41].

Prior to a systematic discussion of the impact of defects on the interfacial water structure, we first discuss another typical interfacial water structure identified in our simulations. In this structure [Figs. 1(c) and 1(d)], water molecules on the $\mathrm{O}_{2 c}$ row mostly form no more than one hydrogen bond with $\mathrm{O}_{2 c}$, in contrast to two hydrogen bonds in the high-density model. In addition, water molecules on $\mathrm{Ti}_{5 c}$ may directly form hydrogen bonds with the $\mathrm{O}_{2 c}$ rather than with adsorbed water molecules [orange molecules in Figs. 1(a) and 1(c)]. In this unconventional structure, fewer water molecules feature in the contact layer and thus we refer to it as low-density interfacial water. ${ }^{2}$ Figure 1(e) further shows that the low-density interfacial water has fewer hydrogen bonds within the contact layer and fewer hydrogen bonds between the water and the surface. Such differences are also shown in the radial distribution function of water hydrogen $\left(\mathrm{H}_{W}\right)$ around $\mathrm{O}_{2 c}$ [Fig. 1(f)]. In the high-density structure, more $\mathrm{H}$ atoms tend to stay bonded to $\mathrm{O}_{2 c}$ sites, while the density of $\mathrm{H}$ atoms is obviously lower in the low-density model.

the lateral density of the basal bilayer of ice Ih by approximately $10 \%$. The high-density interfacial water is named because it has the highest density of interfacial water observed on this surface.

${ }^{2}$ The high-density and low-density water discussed here refer to interfacial water structures with different lateral water density, which are different from the high-density and low-density phases in supercooled bulk water, a topic that is beyond the scope of this study.
Another measure to distinguish the low-density and the high-density interfacial water is the $g(z)$ profile, defining the position distribution of water molecules perpendicular to the interface. Figures 2(b) and 2(c) show all $g(z)$ profiles of ten different simulations, including a pristine substrate and nine substrates with oxygen vacancy, which are labeled from $V_{\mathrm{O}} 1$ to $V_{\mathrm{O}} 9$ according to the depth of the oxygen vacancy. The relative stability of these nine vacancy sites is discussed in Fig. S17 in [41]. In reality, vacancies may migrate across the substrates and have a dynamical influence on the interfacial water structure [23,27]; therefore, as a first step it is important to understand how each vacancy site impacts the interfacial water structure. Figure 2(a) shows a side view of the pristine model; the colored circles highlight the positions of oxygen vacancy in the other nine substrates. Overall, from the $g(z)$ profiles, interfacial water in these ten simulations can be categorized into four high-density [Fig. 2(b)] and six low-density [Fig. 2(c)] structures. For both the high-density and the low-density interfacial water, a sharp peak at about $2 \AA$ corresponds to water adsorbed on $\mathrm{Ti}_{5 c}$. In the high-density model, water molecules bonded to $\mathrm{O}_{2 c}$ sites give rise to a second peak at about $2.6 \AA$, while the peak is missing for the low-density model [gray shaded area in Figs. 2(b) and 2(c)]. An immediate consequence of such structural changes is the density reduction of interfacial water; a quantitative analysis will be discussed later.

Besides the density reduction, the low-density interfacial water is accompanied by a fluxional feature, which is reflected in Figs. 3(a) and 3(b) by projected trajectories of $\mathrm{O}_{W}$ in the $x-y$ plane. There are six black trajectories indicating six water molecules on $\mathrm{Ti}_{5 c}$. Because the energy of water bonding to $\mathrm{Ti}_{5 c}$ is significantly higher than the energy of hydrogen bonding to $\mathrm{O}_{2 c}[47,48]$, these molecules remain adsorbed in all simulations. A difference appears for water molecules on $\mathrm{O}_{2 c}$ sites (viridis lines), whose trajectories are concentrated in the high-density model, while they are extended in the low-density model. Another feature indicated by the trajectory projection is that the high-density interfacial water layer is ordered, whereas the fluxional interfacial water layer of the low-density model is disordered. To quantify the ordering, we define an order parameter $\sigma$ based on the lateral angular distribution of water. The order parameter is calculated by $\sigma=\frac{1}{N} \sum_{i}^{N} \cos (3 \theta)$ in polar coordinate system, where $N$ is the total number of water molecules within a radius of $3 \AA$ from $\mathrm{O}_{2 c}$ and $\theta$ is the polar angle of the molecule (on $\mathrm{Ti}_{5 c}$ )molecule (on $\mathrm{O}_{2 c}$ ) line with respect to the $x$ axis (Fig. $\mathrm{S} 4 \mathrm{a}$ in [41]). Orderly adsorbed water molecules on $\mathrm{Ti}_{5 c}$ and $\mathrm{O}_{2 c}$ sites feature a distribution with $\theta=0, \frac{2}{3} \pi$, and $\frac{4}{3} \pi$, where $\sigma$ attains a maximum value of 1 . In contrast, $\sigma$ has a minimum value of -1 in directions around $\theta=\frac{1}{3} \pi, \pi$, and $\frac{5}{3} \pi$. As a result, for a relatively ordered distribution of water in the high-density model, $\sigma$ approaches 1 , whereas the fluxional distribution in the low-density model results in a smaller, possibly negative, value of $\sigma$.

Furthermore, Fig. 3(c) indicates there is a relation between the density of interfacial water and the order parameter $\sigma$. The number density of water molecules in Fig. 3(c) is defined by the number of water molecules per unit cell within the region of $3 \AA$ above the $\mathrm{Ti}_{5 c}$ surface. Overall, the higher the density is, the larger the order parameter $\sigma$ is, meaning the 
(a)

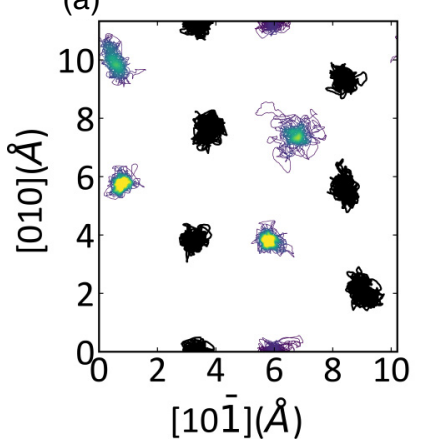

(b)

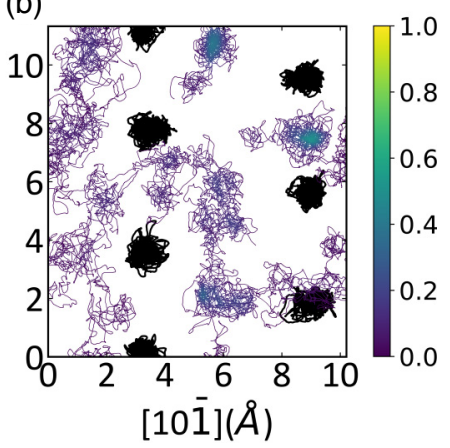

(c)

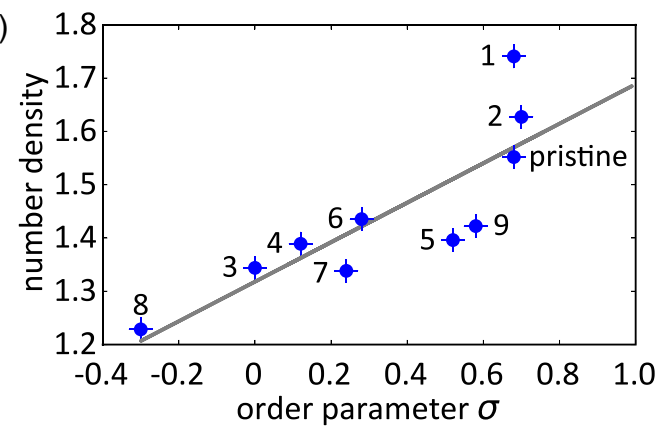

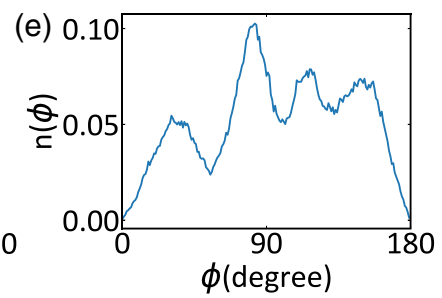

FIG. 3. Fluxional properties. Projected trajectories for (a) the pristine system and (b) $V_{\mathrm{O}} 8$ systems. Figure $\mathrm{S} 7$ in [41] plots projected trajectories for all ten systems. The position of water is identified by the oxygen atom. A uniform sequential colormap of viridis represents the lateral distribution density of a water molecule adsorbed on $\mathrm{O}_{2 c}$. Water molecules on $\mathrm{Ti}_{5 c}$ are plotted with black lines. Water molecules within the first shell, with a cutoff radius of $3 \AA$ from $\mathrm{O}_{2 c}$ and $2.5 \AA$ from $\mathrm{Ti}_{5 c}$, are taken into account. (c) Number density (water molecules bonded to $\mathrm{O}_{2 c}$ ) and order parameter of the ten systems labeled. The gray line is a guide to the eye, showing a positive relation between the density and the ordering parameter. The distribution of water orientation $\phi$ is plotted in (d) the pristine substrate and (e) the $V_{\mathrm{O}} 8$ system. The inset shows the definition of $\phi$, namely, the angle between the surface normal and the orientation of a water molecule.

structure is more ordered. However, when the density is lower the structure is less ordered and more fluxional. The disordering of the contact layer is also reflected on the orientation of water molecules. Figures 3(d) and 3(e) plot the distribution of the molecular orientation $\phi$ of water above the $\mathrm{O}_{2 c}$ rows, which are hydrogen bonded with $\mathrm{O}_{2 c}$ sites directly or with the water adsorbed on $\mathrm{Ti}_{5 c}$. For the ordered high-density model, there is a clear tendency of water molecules to align in a double-leg orientation, which peaks at about $160^{\circ}$ with respect to the surface normal [Fig. 3(d)]. In contrast, a typical fluxional interfacial model would break such a tendency [Fig. 3(e)]. This difference in orientation indicates that the fluxional molecules alternately form donating hydrogen bonds with $\mathrm{O}_{2 c}$ and the bulk liquid. The distribution profiles of all ten systems are shown in the Supplemental Material (Figs. S5 and S6), supporting the analyses discussed. In addition, the fluxional behaviors are accompanied by dynamic exchanges between the contact layer and the bulk liquid (Fig. S8 in [41]).

From the above results, a particularly interesting finding is that the density reduction and the fluxional interfacial water layer are observed on substrates with deep vacancies. Even for the $V_{\mathrm{O}} 7$ system, showing a second peak on $g(z)$ similar to the conventional high-density interfacial water model, other analyses of the order parameter [Fig. 3(c)], water orientation (Fig. S5h in [41]), and number of hydrogen bonded water molecules (Fig. S6h in [41]) clarify its fluxional nature. The vacancy $V_{\mathrm{O}} 9$, located approximately $9 \AA$ under the interfacial water layer, is the deepest vacancy considered in our simulations, and our results suggest that deeper vacancies would have similar effects. This means that only the pristine substrate and the substrates with surface vacancies $\left(V_{\mathrm{O}} 1\right.$ and $\left.V_{\mathrm{O}} 2\right)$ maintain the high-density interfacial water. Given the fact that in $\mathrm{TiO}_{2}$ oxygen vacancies are ubiquitous at different depths across the substrate, low-density fluxional interfacial water may appear generally. The density reduction appearing in our simulation is in line with the experimental results of Nadeem et al., in which they found that a 50\% coverage of water on $\mathrm{O}_{2 c}$ fits best with measurements [46].

It is also worth noting that our results do not contradict previous studies where water dissociation was discussed. Recently, Andrade et al. investigated the spontaneous dissociation of water on the $\mathrm{TiO}_{2}$ surface using machine learning force fields and suggested that spontaneous water dissociation requires nanoseconds for equilibrium [22]. Here we focus on the interfacial structure of water, characterized by the distribution of oxygen positions, whose equilibrium is often reached within a few tens of picoseconds. In fact, water dissociation induced by a surface $V_{\mathrm{O}}$ is also observed in our simulations with $V_{\mathrm{O}} 1$ and $V_{\mathrm{O}} 2$ (Fig. S18 in [41]). Dissociation, however, does not lead to the density reduction of interfacial water. The fact that surface vacancies $\left(V_{\mathrm{O}} 1\right.$ and $\left.V_{\mathrm{O}} 2\right)$ have minor effects on the interfacial water structure is rather counterintuitive because surface oxygen vacancies are reactive to water and lead to the formation of hydroxide groups on the surface. A previous study by Andrade et al. found low-density interfacial water with a high dissociated interfacial model [49]. However, here we find that dissociation is not a necessary factor for low-density interfacial water.

Previous studies have shown that excess electrons can migrate from subsurface to the surface, affecting hydrogen bonding, adsorption, and dissociation of water [13,30,31]. We note that the behavior of excess electrons is quite sensitive to the DFT methods used. For example, excess electrons may localize and show polaronic behavior when DFT $+U$ methods are used. We have also carried out simulations with SCAN-rvv10 and Perdew-Burke-Ernzerhof $+U$ methods, which show consistent results that density reduction can be induced by deep oxygen vacancies (Figs. S3, S9, S10, and S16 in [41]). Despite challenges existing in terms of an accurate description of polarons and their statistical sampling, as well as that the correlation between interfacial water and polarons is yet to be established, these additional tests suggest that 
(a)

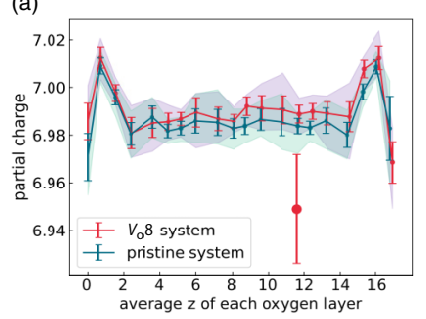

(c)

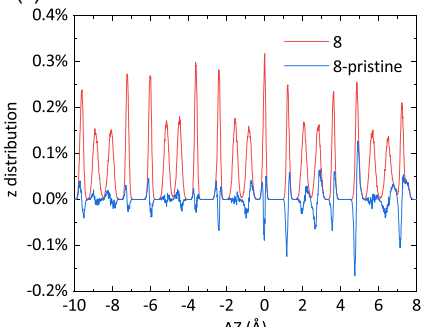

(e)

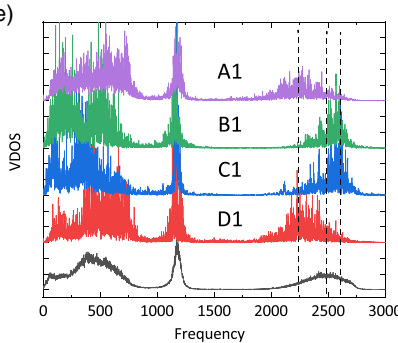

(b)

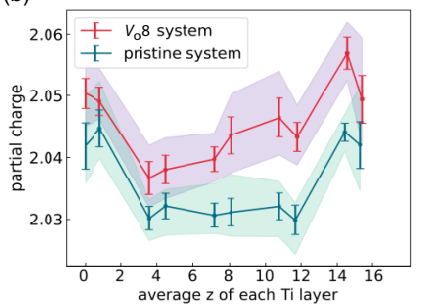

(d)

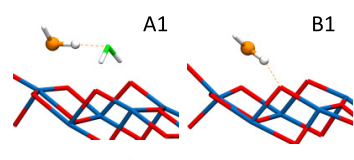

D1
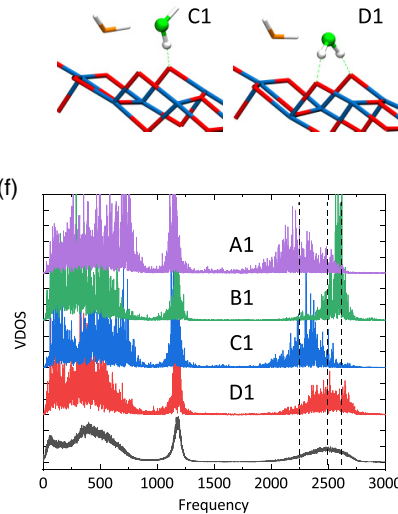

FIG. 4. Substrate atomic position distribution, substrate charge distribution, and VDOS of interfacial water. The average partial charge of every (a) oxygen and (b) titanium substrate layer is plotted. Error bars and shadow regions indicate the standard deviation and the interval between the minimum and maximum, respectively. The average is calculated every 1000 steps and every six atoms in a substrate layer (10 layers for Ti and 20 layers for $\mathrm{O}$ ). The reference zero point of the $z$ coordinate is chosen to be the average $z$ value of atoms in the lowermost interfacial layer. (c) The red line is the distribution density of oxygen atoms (20 layers) except for the dangling oxygen atom in the $V_{\mathrm{O}} 8$ system. The blue line is the difference from the same distribution density in the pristine system, showing that the difference in two substrates is extended across the whole substrate. Here $\Delta Z$ is defined as the difference between the $z$ value of an oxygen atom and the average $z$ value of all six oxygen atoms in the ninth layer. The ninth oxygen layer is chosen as the reference for the plot, since its average oxygen position is unaffected by the existence of $V_{\mathrm{O}}$. (d) Snapshots A1-D1 show the structures concerned in the VDOS of (e) the pristine system and (f) the $V_{\mathrm{O}} 8$ system. Black lines in the VDOS represent the liquid reference in the systems.

low-density fluxional interfacial water is not an artifact and not sensitive to the description of localized electrons and the extent of water dissociation.

To provide deeper physical insights into the cause of lowdensity interfacial water, we further compared the position and charge distribution of the $V_{\mathrm{O}} 8$ system and the pristine substrate. Figure 4(a) and 4(b) plot the Bader charge of oxygen and titanium atoms on each layer across the pristine and $V_{\mathrm{O}} 8$ substrates. In comparison to the pristine system, oxygen vacancies result in a few percent difference of the average charge, while the substrates remain insulating (Fig. S19 in [41]). Based on an electrostatic interaction estimate,

this magnitude of change may induce an electrostatic energy difference of tens of meV. Such a difference is minor in chemical reactions, but it is large enough to alter the hydrogen bonding network, thus leading to a new interfacial water structure on $\mathrm{TiO}_{2}$. For example, previous studies on anatase surface have shown a competition between two water molecules for hydrogen bonding to the bridging oxygen that raises the adsorption energy by about $33 \mathrm{meV}$ [50]. It was also shown that the surface charges can have significant effects on the structure of water on metal surfaces [51]. In the same spirit, a slight electron deficit on bridging oxygen [Fig. 4(a)] in a defective system leads to weaker hydrogen bonding, hence pushing one of the competing water molecules away from the bridging oxygen.

Moreover, Fig. 4(c) plots the oxygen atom distribution and their difference in the $V_{\mathrm{O}} 8$ and pristine systems; the interfacial water structure on these two substrates are quite different, displaying a collective variation of atomic positions across the substrate layers. The collective variation is a typical elastic effect that can physically transfer interactions to a long range. Similar effects have been observed in rutile, where water adsorption and dissociation energies vary along with the substrate positions [52]. In the Supplemental Material we further compare the $V_{\mathrm{O}} 5$ and $V_{\mathrm{O}} 8$ systems. These two systems have oxygen vacancies located on the opposite sides of an interspace and during the simulations the oxygen atom vibrates in the middle, resulting in two very similar substrates structurally (Fig. S11 in [41]) and electronically (Fig. S12 in [41]). Yet minor changes in atomic position around the vacancy lead to obvious differences in the interfacial water structure.

Above we have discussed a few analyses related to the cause of interfacial water density reduction, including chemical, polaronic, electrostatic, and positional changes. It seems that none of them is a dominating factor and the interfacial water density reduction may be a result of the balance of these factors together. The delicate nature also indicates that experimental identification of the interfacial water structure on the anatase (101)-water interface is a great challenge, requiring not only advanced characterization techniques but also clean and well-controlled substrate samples. The interfacial water density change reflects to some extent on the hydrogen bonding change at the interface, which can be detected experimentally using vibrational spectroscopies. Figures 4(e) and 4(f) show the vibrational density of state (VDOS) from $a b$ initio molecular dynamics trajectories of the pristine and $V_{\mathrm{O}} 8$ systems. The VDOS profiles A1-D1 in Figs. 4(e) and 4(f) correspond to the donor O-H stretching highlighted in Fig. 4(d). For comparison, the VDOSs from water molecules are shown as black lines. The red and blue shifts in the stretching mode indicate strong and weak hydrogen bonds. The strength difference in $\mathrm{C} 1$ and $\mathrm{D} 1$ between pristine and defective systems leads to the difference in the interfacial structure. Specifically, the oxygen vacancy shifts the tendency of the local hydrogen bonding structure from $\mathrm{D} 1$ to $\mathrm{C} 1$, which is also highlighted in the dipole orientation profiles [Figs. 3(d) and 4(e)].

Finally, to examine whether our findings can extend to other systems, we have considered a few other substrates, namely, rutile (110), anatase (001), and silica (0001) (Figs. S13 and S14 in [41]). These substrates cover three 
different kinds: (i) On rutile (110) water remains intact, (ii) on anatase (001) water dissociates, and on silica (0001) the surface is readily hydroxylated. In the end, on these substrates tested we do not see a strong influence of deep oxygen vacancies on the interfacial water structure. Therefore, whether there are other substrates that possess similar interesting behaviors identified on anatase (101) remains an open question.

In summary, we have reported $a b$ initio simulations of the water-anatase (101) interface, where we have identified the low-density fluxional interfacial water due to oxygen vacancies in the substrate. We showed that the influence of oxygen vacancies can be rather long range and indirect, which led to the finding that even the deepest vacancy considered in our simulations can strongly impact the interfacial water structure. We also revealed that the density reduction and induced flexibility are closely related and the behaviors are likely to be physically amplified by minor changes in the substrate due to the presence of oxygen vacancies. The nontrivial long-range effect identified in our study is another highlight of the complexity of interfacial water structure and it is also potentially an opportunity for materials engineering to manipulate interfacial water properties by controlling the inner part of materials.

The authors thank Angelos Michaelides for helpful discussions. This work was supported by the National Natural Science Foundation of China under Grant No. 11974024 and the Strategic Priority Research Program of Chinese Academy of Sciences under Grant No. XDB33000000. We are grateful for computational resources provided by Peking University, the TianHe-1A supercomputer, Shanghai Supercomputer Center, and Songshan Lake Materials Laboratory.
[1] A. Hodgson and S. Haq, Water adsorption and the wetting of metal surfaces, Surf. Sci. Rep. 64, 381 (2009).

[2] O. Björneholm, M. H. Hansen, A. Hodgson, L.-M. Liu, D. T. Limmer, A. Michaelides, P. Pedevilla, J. Rossmeisl, H. Shen, G. Tocci, E. Tyrode, M.-M. Walz, J. Werner, and H. Bluhm, Water at interfaces, Chem. Rev. 116, 7698 (2016).

[3] P. G. Debenedetti and M. L. Klein, Chemical physics of water, Proc. Natl. Acad. Sci. USA 114, 13325 (2017).

[4] H. Kuhlenbeck, S. Shaikhutdinov, and H.-J. Freund, Wellordered transition metal oxide layers in model catalysis - A series of case studies, Chem. Rev. 113, 3986 (2013).

[5] G. C. Sosso, J. Chen, S. J. Cox, M. Fitzner, P. Pedevilla, A. Zen, and A. Michaelides, Crystal nucleation in liquids: Open questions and future challenges in molecular dynamics simulations, Chem. Rev. 116, 7078 (2016).

[6] A. Striolo, A. Michaelides, and L. Joly, The carbon-water interface: Modeling challenges and opportunities for the water-energy nexus, Annu. Rev. Chem. Biomol. Eng. 7, 533 (2016).

[7] M. Fitzner, P. Pedevilla, and A. Michaelides, Predicting heterogeneous ice nucleation with a data-driven approach, Nat. Commun. 11, 4777 (2020).

[8] M. Sulpizi, M.-P. Gaigeot, and M. Sprik, The silica water interface: How the silanols determine the surface acidity and modulate the water properties, J. Chem. Theory Comput. 8, 1037 (2012)

[9] J. A. Kattirtzi, D. T. Limmer, and A. P. Willard, Microscopic dynamics of charge separation at the aqueous electrochemical interface, Proc. Natl. Acad. Sci. USA 114, 13374 (2017).

[10] O. M. Magnussen and A. Gross, Toward an atomic-scale understanding of electrochemical interface structure and dynamics, J. Am. Chem. Soc. 141, 4777 (2019).

[11] C. Zhang, J. Hutter, and M. Sprik, Coupling of surface chemistry and electric double layer at $\mathrm{TiO}_{2}$ electrochemical interfaces, J. Phys. Chem. Lett. 10, 3871 (2019).

[12] F. De Angelis, C. Di Valentin, S. Fantacci, A. Vittadini, and A. Selloni, Theoretical studies on anatase and less common $\mathrm{TiO}_{2}$ phases: Bulk, surfaces, and nanomaterials, Chem. Rev. 114, 9708 (2014).
[13] S. Selcuk and A. Selloni, Facet-dependent trapping and dynamics of excess electrons at anatase $\mathrm{TiO}_{2}$ surfaces and aqueous interfaces, Nat. Mater. 15, 1107 (2016).

[14] C. N. R. Rao and S. Dey, Solar thermochemical splitting of water to generate hydrogen, Proc. Natl. Acad. Sci. USA 114, 13385 (2017)

[15] G. Tocci, M. Bilichenko, L. Joly, and M. Iannuzzi, Ab initio nanofluidics: Disentangling the role of the energy landscape and of density correlations on liquid/solid friction, Nanoscale $\mathbf{1 2}$ 10994 (2020)

[16] B. C. Stipe, M. A. Rezaei, and W. Ho, Single-molecule vibrational spectroscopy and microscopy, Science 280, 1732 (1998).

[17] M. A. Henderson, The interaction of water with solid surfaces: Fundamental aspects revisited, Surf. Sci. Rep. 46, 1 (2002).

[18] Y. R. Shen and V. Ostroverkhov, Sum-frequency vibrational spectroscopy on water interfaces: Polar orientation of water molecules at interfaces, Chem. Rev. 106, 1140 (2006).

[19] C.-Y. Li, J.-B. Le, Y.-H. Wang, S. Chen, Z.-L. Yang, J.-F. Li, J. Cheng, and Z.-Q. Tian, In situ probing electrified interfacial water structures at atomically flat surfaces, Nat. Mater. 18, 697 (2019).

[20] J. Carrasco, A. Hodgson, and A. Michaelides, A molecular perspective of water at metal interfaces, Nat. Mater. 11, 667 (2012).

[21] J. Cheng, X. Liu, J. VandeVondele, M. Sulpizi, and M. Sprik, Redox potentials and acidity constants from density functional theory based molecular dynamics, Acc. Chem. Res. 47, 3522 (2014).

[22] M. F. C. Andrade, H.-Y. Ko, L. Zhang, R. Car, and A. Selloni, Free energy of proton transfer at the water $\mathrm{TiO}_{2}$ interface from $a b$ initio deep potential molecular dynamics, Chem. Sci. 11, 2335 (2020).

[23] U. Aschauer and A. Selloni, Structure of the Rutile $\mathrm{TiO}_{2}(011)$ Surface in an Aqueous Environment, Phys. Rev. Lett. 106, 166102 (2011)

[24] C. L. Pang, R. Lindsay, and G. Thornton, Structure of clean and adsorbate-covered single-crystal rutile $\mathrm{TiO}_{2}$ surfaces, Chem. Rev. 113, 3887 (2013). 
[25] U. Diebold, Perspective: A controversial benchmark system for water-oxide interfaces: $\mathrm{H}_{2} \mathrm{O} / \mathrm{TiO}_{2}(110)$, J. Chem. Phys. 147, 040901 (2017).

[26] O. Bikondoa, C. L. Pang, R. Ithnin, C. A. Muryn, H. Onishi, and G. Thornton, Direct visualization of defect-mediated dissociation of water on $\mathrm{TiO}_{2}(110)$, Nat. Mater. 5, 189 (2006).

[27] Y. Li and Y. Gao, Interplay Between Water and $\mathrm{TiO}_{2}$ Anatase (101) Surface with Subsurface Oxygen Vacancy, Phys. Rev. Lett. 112, 206101 (2014).

[28] N. A. Deskins, G. A. Kimmel, and N. G. Petrik, Observation of molecular hydrogen produced from bridging hydroxyls on anatase $\mathrm{TiO}_{2}$ (101), J. Phys. Chem. Lett. 11, 9289 (2020).

[29] N. A. Deskins, R. Rousseau, and M. Dupuis, Defining the role of excess electrons in the surface chemistry of $\mathrm{TiO}_{2}$, J. Phys. Chem. C 114, 5891 (2010).

[30] C. M. Yim, J. Chen, Y. Zhang, B.-J. Shaw, C. L. Pang, D. C. Grinter, H. Bluhm, M. Salmeron, C. A. Muryn, A. Michaelides, and $\mathrm{G}$. Thornton, Visualization of water-induced surface segregation of polarons on rutile $\mathrm{TiO}_{2}(110)$, J. Phys. Chem. Lett. 9, 4865 (2018).

[31] J. Chen, C. Penschke, A. Alavi, and A. Michaelides, Small polarons and the Janus nature of $\mathrm{TiO}_{2}(110)$, Phys. Rev. B 101, 115402 (2020).

[32] R. Car and M. Parrinello, Unified Approach for Molecular Dynamics and Density-Functional Theory, Phys. Rev. Lett. 55, 2471 (1985).

[33] G. Kresse and J. Furthmüller, Efficient iterative schemes for ab initio total-energy calculations using a plane-wave basis set, Phys. Rev. B 54, 11169 (1996).

[34] J. Klimeš, D. R. Bowler, and A. Michaelides, Chemical accuracy for the van der Waals density functional, J. Phys.: Condens. Matter 22, 022201 (2010).

[35] J. Klimeš, D. R. Bowler, and A. Michaelides, Van der Waals density functionals applied to solids, Phys. Rev. B 83, 195131 (2011).

[36] J. Sun, A. Ruzsinszky, and J. P. Perdew, Strongly Constrained and Appropriately Normed Semilocal Density Functional, Phys. Rev. Lett. 115, 036402 (2015).

[37] H. Peng, Z.-H. Yang, J. P. Perdew, and J. Sun, Versatile van der Waals Density Functional Based on a Meta-Generalized Gradient Approximation, Phys. Rev. X 6, 041005 (2016).

[38] S. L. Dudarev, G. A. Botton, S. Y. Savrasov, C. J. Humphreys, and A. P. Sutton, Electron-energy-loss spectra and the structural stability of nickel oxide: An LSDA+U study, Phys. Rev. B 57, 1505 (1998).

[39] G. Kresse and D. Joubert, From ultrasoft pseudopotentials to the projector augmented-wave method, Phys. Rev. B 59, 1758 (1999).
[40] M. Setvin, C. Franchini, X. Hao, M. Schmid, A. Janotti, M. Kaltak, C. G. Van de Walle, G. Kresse, and U. Diebold, Direct View at Excess Electrons in $\mathrm{TiO}_{2}$ Rutile and Anatase, Phys. Rev. Lett. 113, 086402 (2014).

[41] See Supplemental Material at http://link.aps.org/supplemental/ 10.1103/PhysRevResearch.3.L042014 for additional data, convergence tests, computational details, and extended analyses.

[42] A. Tilocca and A. Selloni, DFT-GGA and DFT $+U$ simulations of thin water layers on reduced $\mathrm{TiO}_{2}$ anatase, J. Phys. Chem. C 116, 9114 (2012).

[43] Z. Zhao, Z. Li, and Z. Zou, Structure and properties of water on the anatase $\mathrm{TiO}_{2}(101)$ surface: From single-molecule adsorption to interface formation, J. Phys. Chem. C 116, 11054 (2012).

[44] U. J. Aschauer, A. Tilocca, and A. Selloni, Ab initio simulations of the structure of thin water layers on defective anatase $\mathrm{TiO}_{2}(101)$ surfaces, Int. J. Quantum Chem. 115, 1250 (2015).

[45] D. Selli, G. Fazio, G. Seifert, and C. Di Valentin, Water multilayers on $\mathrm{TiO}_{2}$ (101) anatase surface: Assessment of a DFTB-based method, J. Chem. Theory Comput. 13, 3862 (2017).

[46] I. M. Nadeem, J. P. W. Treacy, S. Selcuk, X. Torrelles, H. Hussain, A. Wilson, D. C. Grinter, G. Cabailh, O. Bikondoa, C. Nicklin, and A. Selloni, Water dissociates at the aqueous interface with reduced anatase $\mathrm{TiO}_{2}$ (101), J. Phys. Chem. Lett. 9, 3131 (2018)

[47] G. S. Herman, Z. Dohnálek, N. Ruzycki, and U. Diebold, Experimental investigation of the interaction of water and methanol with anatase-TiO 2 (101), J. Phys. Chem. B 107, 2788 (2003).

[48] A. Dahal and Z. Dohnálek, Formation of metastable water chains on anatase $\mathrm{TiO}_{2}(101)$, J. Phys. Chem. C 121, 20413 (2017).

[49] M. F. Calegari Andrade, H.-Y. Ko, R. Car, and A. Selloni, Structure, polarization, and sum frequency generation spectrum of interfacial water on anatase $\mathrm{TiO}_{2}$, J. Phys. Chem. Lett. 9, 6716 (2018).

[50] Y. He, A. Tilocca, O. Dulub, A. Selloni, and U. Diebold, Local ordering and electronic signatures of submonolayer water on anatase $\mathrm{TiO}_{2}(101)$, Nat. Mater. 8, 585 (2009).

[51] M. Otani, I. Hamada, O. Sugino, Y. Morikawa, Y. Okamoto, and T. Ikeshoji, Structure of the water/platinum interface-A first principles simulation under bias potential, Phys. Chem. Chem. Phys. 10, 3609 (2008).

[52] L.-M. Liu, C. Zhang, G. Thornton, and A. Michaelides, Structure and dynamics of liquid water on rutile $\mathrm{TiO}_{2}(110)$, Phys. Rev. B 82, 161415(R) (2010). 